\title{
Long lived central engines in Gamma Ray Bursts
}

\author{
Gabriele Ghisellini
}

INAF - Osservatorio Astronomico di Brera, Merate, Italy

\begin{abstract}
The central engine of Gamma Ray Bursts may live much longer than the duration of the prompt emission. Some evidence of it comes from the presence of strong precursors, post-cursors, and X-ray flares in a sizable fraction of bursts. Additional evidence comes from the fact that often the X-ray and the optical afterglow light curves do not track one another, suggesting that they are two different emission components. The typical "steep-flat-steep" behavior of the X-ray light curve can be explained if the same central engine responsible for the main prompt emission continues to be active for a long time, but with a decreasing power. The early X-ray "afterglow" emission is then the extension of the prompt emission, originating at approximately the same location, and is not due to forward shocks. If the bulk Lorentz factor $\Gamma$ is decreasing in time, the break ending the shallow phase can be explained, since at early times $\Gamma$ is large, and we see only a fraction of the emitting area. Later, when $\Gamma$ decreases, we see an increasing fraction of the emitting surface up to the time when $\Gamma \sim 1 / \theta_{\mathrm{j}}$. This time ends the shallow phase of the $\mathrm{X}$-ray light curve. The origin of the late prompt emission can be the accretion of the fall-back material, with an accretion rate $\dot{M} \propto t^{-5 / 3}$. The combination of this late prompt emission with the flux produced by the standard forward shock can explain the great diversity of the optical and the X-ray light curves.
\end{abstract}

Keywords: Gamma Ray Bursts, Afterglow, Prompt emission

PACS: $98.70 . \mathrm{Rz}$, 95.85.Pw, 95.85.Nv,95.30.Gv

\section{INTRODUCTION}

The X-ray light curves, as observed by Swift, have shown a complexity unforeseen before. Besides the "normal" behavior, as observed by BeppoSAX after several hours from the trigger, we now know that a good fraction of GRBs show a steep decay soon after the end of the burst as seen by BAT, followed by a plateau lasting for a few thousands of seconds, ending at the time $T_{A}$ (following [17]). This behavior, named "Steep-Flat-Steep" [15, 11] has been interpreted in several ways (for reviews, see e.g. [18]) none of which seems conclusive. Furthermore, in nearly half of the bursts, we observe X-ray flares, of relatively short duration $\Delta t$ (i.e. $\Delta t / t \sim 0.1$, see [3]) occurring even several hours after the trigger. Considering X-ray flares in different GRBs, [9] have shown that their average luminosity goes like $t^{-5 / 3}$, the same time decay of the accretion rate of the fall-back material (see [2]; [19]).

The optical light curves are also complex, but rarely track the behavior of the X-ray flux (see e.g. [13, 14]), suggesting a possible different origin. At the other time extreme, for $10-15 \%$ of the bursts, we observe precursor emission, separated from the main event, in some cases, by hundreds of seconds. The energy contained in these precursors is comparable to the energy of the main event, and the spectrum is indistinguishable [1], suggesting that they are produced by the same engine producing the main event.

To explain the "steep-flat-steep" X-tray light curves, [16] and [4] (see also Daigne, these proceedings) suggested that the $\mathrm{X}$-ray plateau emission is not due to the forward, 


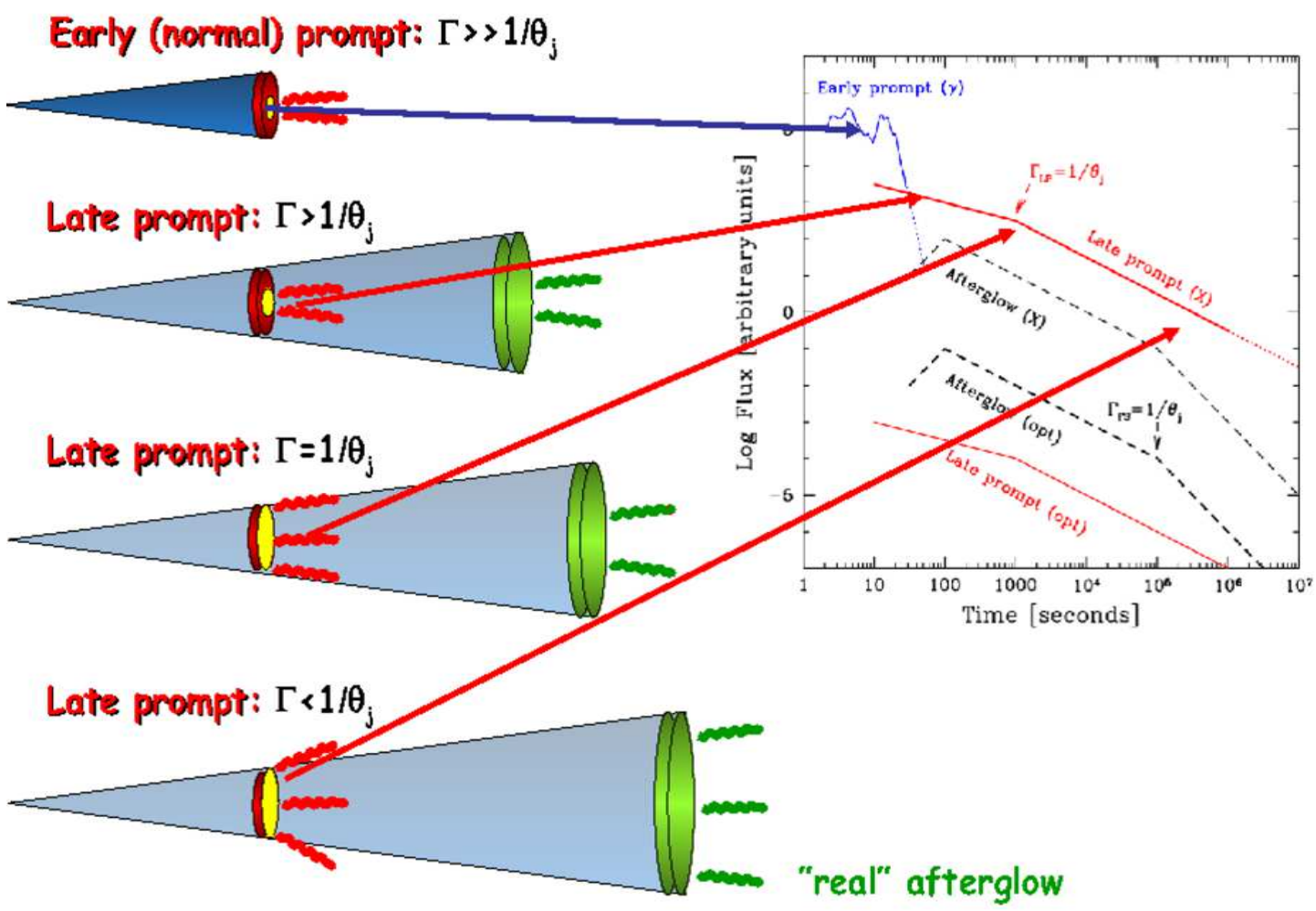

FIGURE 1. Cartoon of the proposed model, and schematic illustration of the different components contributing to the $\mathrm{X}$-ray and optical light curves, as labeled. Scales are arbitrary. The early prompt phase is erratic, with shells of varying $\Gamma$ and power. Then the central engine produces shells of progressively less power and bulk Lorentz factors, producing a smoother light curve. Since the average $\Gamma$-factor is decreasing, the observer sees an increasing portion of the emitting area, until all of it becomes visible when $\Gamma \sim 1 / \theta_{j}$. When this occurs there is a break in the light curve, associated with the ending of the shallow phase. The case illustrated here is only one (likely the most common) possible case, when the $\mathrm{X}-$ ray flux is dominated by late prompt emission (solid line, the dotted line corresponds to an extrapolation at very late times), while the optical flux is dominated by the real afterglow (dashed). Adapted from [6].

but to the reverse shock running into ejecta of relatively small (and decreasing) Lorentz factors. This however requires an appropriate $\Gamma$-distribution of the ejecta, and also the suppression of the $\mathrm{X}$-ray flux produced by the forward shock.

We [6] instead suggested that the plateau phase of the X-ray (and sometimes of the optical) emission is due to a prolonged activity of the central engine (see also [8]), responsible for a "late-prompt" phase: after the early "standard" prompt phase the central engine continues to produce for a long time (i.e. days) shells of progressively lower power and bulk Lorentz factor. The dissipation process during this and the early phases occur at similar radii (namely close to the transparency radius). The reason for the shallow decay phase, and for the break ending it, is that the $\Gamma$-factors of the late shells are monotonically decreasing, allowing to see an increasing portion of the emitting surface, until all of it is visible. Then the break occurs when $\Gamma(t)=1 / \theta_{j}$. 


\section{THE LATE PROMPT EMISSION SCENARIO}

Willingale et al. [17] have proposed to describe the X-ray afterglow light curve with a rising exponential connecting to a power law function. The end of the shallow phase is the junction between the exponential and the power law, and it is called $T_{A}$.

Investigating the possibility that $T_{A}$ might be a jet break, as suggested by [17], we [10] demonstrated that it is not, yet it may be produced by a mechanism similar to the process responsible for the jet break visible during the deceleration of the fireball. Suppose that the accretion onto the newly formed black hole occurs in two phases: the first is short, intense, erratic, corresponding to the early prompt phase of GRBs. The accreting matter may be the equatorial core material which failed to form the black hole in the first place. Being very dense, it can sustain a strong magnetic field, which in turn efficiently extracts the rotational energy of the black hole. The second phase is longer, smoother, with a rate decreasing in time, corresponding to the late prompt emission. The accreting matter may be fall-back material, with a density smaller than in the early phases. The magnetic field that this matter can sustain is weaker than before, with a corresponding smaller power extracted from the black hole spin. This may well correspond to production of shells of smaller $\Gamma$-factors. These shells can dissipate part of their energy with the same mechanism of the early ones. Occasionally, in this late prompt phase, the central engine may produce a faster than average shell, originating the late flares often observed in the Swift/XRT light curves.

In this scenario there is a simple relation between time profile of $\Gamma$ and the observed decay slopes before and after $T_{A}$. The plateau phase is described by $L(t) \propto t^{-\alpha_{2}}$, followed by a steeper decay $L(t) \propto t^{-\alpha_{3}}$. Then, by geometry alone, [6] derived:

$$
\Gamma \propto t^{-\left(\alpha_{3}-\alpha_{2}\right) / 2}
$$

By setting $L(t)=\eta \Gamma \dot{M}_{\text {out }} c^{2} \propto t^{-\alpha_{3}}$ (after $T_{A}$ we see the entire jet surface) we get:

$$
\dot{M}_{\text {out }} \propto t^{-\left(\alpha_{2}+\alpha_{3}\right) / 2}
$$

Since $\alpha_{3}>1$, the total energy involved in the late prompt emission is modest, at most comparable with the entire early prompt energy.

\section{A UNIFYING VIEW}

The late prompt emission scenario adds one component, it does not substitute the forward shock emission with something else. Both mechanisms are working, both making some X-ray and optical flux. We may then have a variety of cases: both the optical and the X-rays can be late prompt emission or forward shock emission; or X-rays and optical can be "decoupled", one due to late prompt and the other to the forward shock (that hereafter we call "real afterglow").

We [7] have then tested these ideas collecting all X-ray and optical light-curves of Swift bursts of known redshift and optical extinction (at the host). This ensures that we can construct reliable luminosity vs rest frame time profiles for both bands. Fig. 2] shows all the X-ray light-curves in the rest frame. Overall, they appear to decay roughly as 


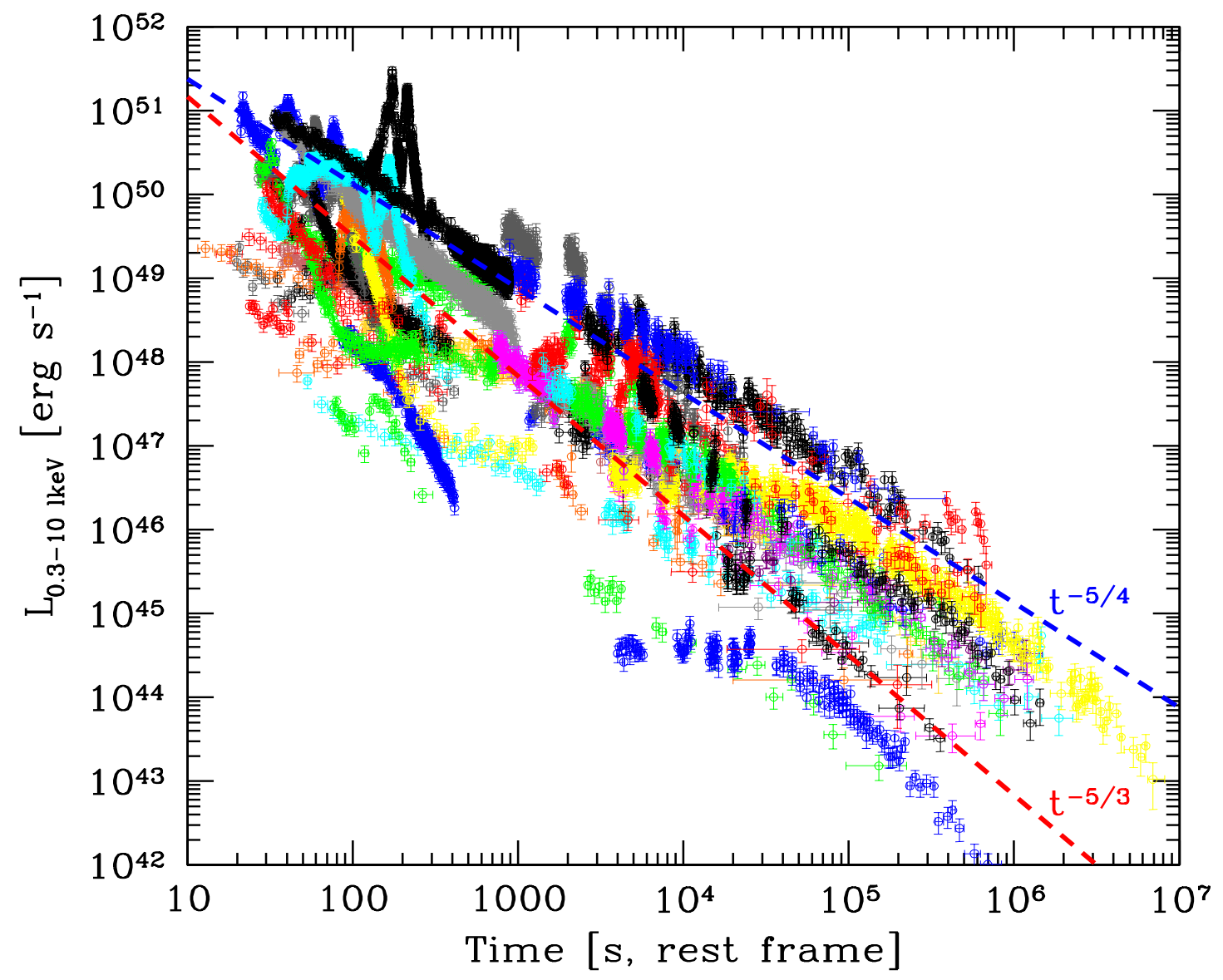

FIGURE 2. The X-ray light curve (in the $0.3-10 \mathrm{keV}$ band) for a sample of 33 Swift GRBs with redshift and measured extinction on the optical. The two dashed line correspond to $t^{-5 / 4}$ and $t^{-5 / 3}$ : the latter appears to be too steep to explain the observed general behavior. But when the light curves are de-convolved into the late prompt and "real" afterglow components, we recover $t^{-5 / 3}$ for the late prompt emission after the end of the shallow phase.

$t^{-5 / 4}$ (see the appropriate dashed line). This is slope is flatter than $t^{-5 / 3}$, expected if the the $\mathrm{X}$-ray luminosity is proportional to the the accretion rate of the fall-back material (of the massive exploding star) onto the black hole ([2], [19]).

For all the 33 GRBs we tried to model both the X-ray and the optical light curve with the sum of two components. The first is the standard forward shock emission, calculated following the prescription of [12], the second mimics the late prompt emission through a phenomenological parametrization. We have then doubled the number of input parameters ( 6 for the forward shock, 7 for the "late prompt"), and the fact that we obtain a good representation of the data may come not as a surprise. However, several parameters of 

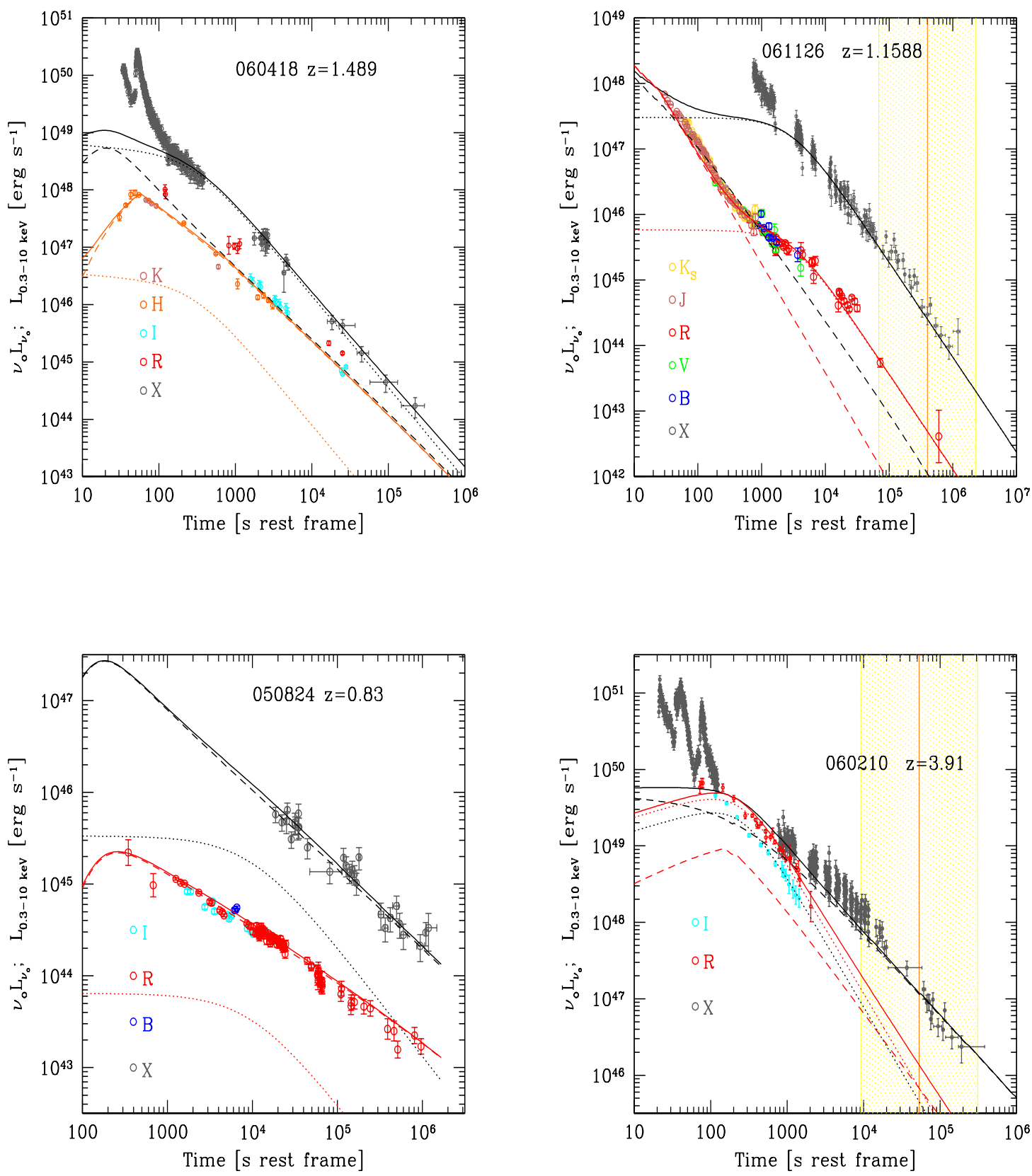

FIGURE 3. Some examples of $\mathrm{X}-$ ray $[0.3-10 \mathrm{keV}]$ and optical $\left(v L_{V}\right)$ light-curves, calculated in the rest frame. The optical and $\mathrm{X}$-ray luminosities have been de-absorbed and $\mathrm{K}$-corrected. The vertical line (and shaded band) is the rest frame jet break time (and its $3 \sigma$ uncertainty) expected if the burst obeys the Ghirlanda relation [5]. The dashed and dotted lines correspond to "real" afterglow and late prompt emission, respectively. Solid lines correspond to the sum. In GRB 060418 (representative of the most common case), the late prompt process dominates the X-ray luminosity, and the real afterglow dominates the optical one. In GRB 061126, instead, the optical at early times is dominated by the real afterglow, but becomes late prompt emission at later times. For GRB 050824 the real afterglow emission dominates in both bands. Finally, in GRB 060210 we have that the real afterglow dominates the X-ray luminosity, while the late prompt is important in the optical band, at least for the plotted (early) times (from [7]). 
the phenomenological (late prompt) part are really well constrained, such as the time $T_{A}$, the time decay slopes before and after $T_{A}$, and the X-ray frequency spectrum. We obtain in all cases a good representation. Fig. 3 shows 4 examples, selected to show 4 different cases. GRB 060418 illustrates the most common case: the late prompt process dominates the X-ray luminosity, and the "real afterglow" dominates the optical one. In GRB 061126, instead, the optical at early times is dominated by the real afterglow, but becomes late prompt emission at later times. For GRB 050824 the real afterglow emission dominates in both bands. Finally, in GRB 060210 we have that the real afterglow dominates the X-ray luminosity, while the late prompt is important in the early optical band (where we have data). The main outcomes of this study are: i) we can explain all the X-ray and optical light-curves within a relatively simple scenario; ii) we can understand why it is difficult to have achromatic jet breaks (but sometimes we do); iii) even when we can see the jet break, the flux after the jet break time can decay in a shallower way than predicted (the unbroken late prompt emission can still contribute); iv) the decay slope of the X-ray late prompt emission can indeed be $5 / 3$ even if we observe a flatter slope, since at relatively late times the real afterglow emission helps in flattening the light curve.

\section{ACKNOWLEDGMENTS}

I gratefully thank all my collaborators: D. Burlon, A. Celotti, C. Firmani, G. Ghirlanda, D. Lazzati, M. Nardini, L. Nava and F. Tavecchio.

\section{REFERENCES}

1. D. Burlon, G. Ghirlanda, G. Ghisellini et al., 2008, ApJ, in press (astro-ph/0806.3076)

2. R.A. Chevalier, 1989, ApJ, 346, 847

3. G. Chincarini, A. Moretti, P. Romano et al., 2007, ApJ, 671, 1903

4. F. Genet, F. Daigne, \& R. Mochkovitch, 2007, MNRAS, 381, 732

5. G. Ghirlanda, G. Ghisellini \& D, Lazzati, 2004, ApJ, 616, 331

6. G. Ghisellini, G. Ghirlanda, L. Nava \& C. Firmani, 2007, ApJ, 658, L75

7. G. Ghisellini, M. Nardini \& G. Ghirlanda, in preparation

8. D. Lazzati \& R. Perna, 2007, MNRAS, 375, L46

9. D. Lazzati, R. Perna \& M.C. Begelman, 2008, MNRAS, 388, L15

10. L. Nava, G. Ghisellini, G. Ghirlanda et al., 2007, MNRAS, 377, 1464

11. J.A. Nousek, C. Kouveliotou, D. Grupe, et al., 2005, ApJ, 642, 389

12. A. Panaitescu \& P. Kumar, 2000, ApJ, 543, 66

13. A. Panaitescu, P. Meszaros, D. Burrows, N. Nousek et al. 2006, MNRAS, 369, 2059

14. A. Panaitescu, 2007, Il Nuovo Cimento, in press (astro-ph/0607396)

15. G. Tagliaferri, M. Goad, G. Chincarini, et al., 2005, Nature, 436, 985

16. L.Z. Uhm \& A.M. Beloborodov, 2007, ApJ, 665, L93

17. R. Willingale, P.T. O'Brien, J.P. Osborne, et al., 2007, ApJ, 662, 1093

18. B. Zhang, 2007, Advances in Space Research, 40, Issue 8, p. 1186 (astro-ph/0611774)

19. W. Zhang, S.E. Woosley \& A. Heger, 2007, ApJ, 679, 639 